\title{
Clinicopathological characteristics and prognostic value of the cancer stem cell marker ALDHI in ovarian cancer: a meta-analysis
}

This article was published in the following Dove Press journal: OncoTargets and Therapy

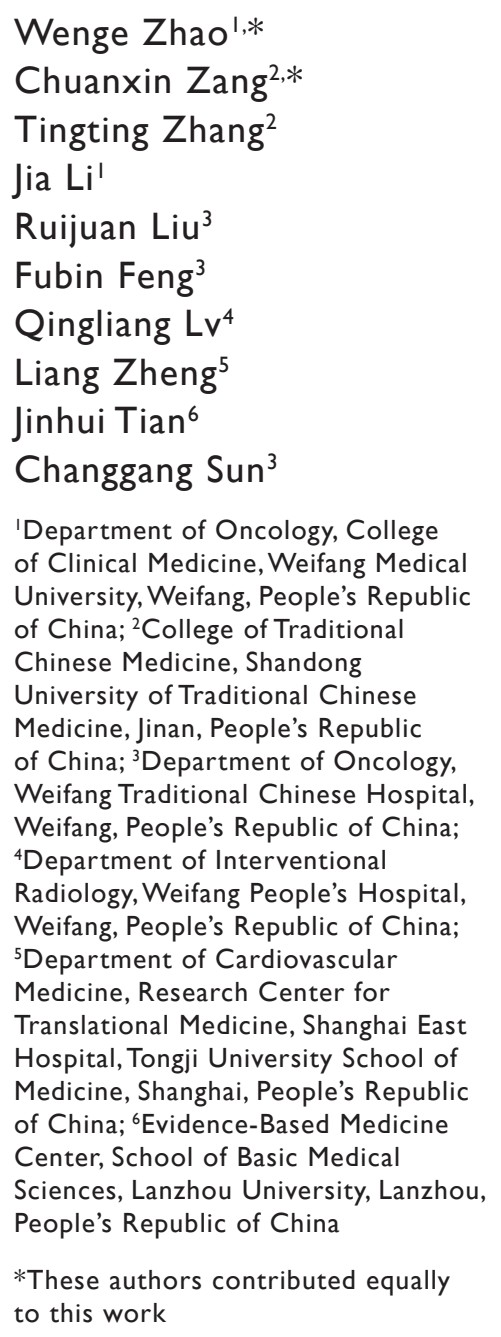

Correspondence: Changgang Sun Department of Oncology, College of Clinical Medicine, Weifang Medical University, No 7166, Baotong West Street, Weifang, Shandong 261053, People's Republic of China Tel +86 I3583690699 Email scgdoctor@।26.com
Background: The clinicopathological and prognostic values of the cancer stem cell marker aldehyde dehydrogenase 1 (ALDH1) in ovarian cancer (OC) remain unknown. The aim of our meta-analysis was to evaluate ALDH1's association with clinicopathological characteristics and its prognostic significance in patients with OC.

Materials and methods: PubMed, Embase, and China Biology Medicine were systematically searched for eligible studies (up to October 2017). Pooled odds ratios (ORs) or hazard ratios (HRs) with 95\% CIs were used to evaluate the association of ALDH1 expression with clinicopathological features and survival outcomes.

Results: A total of 17 papers (18 studies) that included 2,531 patients with OC were analyzed. The results showed a significant association between increasing ALDH1 expression and International Federation of Gynecology and Obstetrics stage (OR 2.02, 95\% CI 1.16-3.52), lymph node metastasis (OR 1.91, 95\% CI 1.01-3.61), and distant metastasis (OR 5.43, 95\% CI 1.44-20.42) in OC. However, no significant correlation was found between increasing ALDH1 expression and age (OR 0.90, 95\% CI 0.25-3.28), tumor size (OR 1.13, 95\% CI $0.75-1.71$ ), tumor location (OR $0.69,95 \%$ CI $0.22-2.13$ ), ascite status (OR $0.74,95 \%$ CI 0.49-1.11), resistance status (OR 0.70, 95\% CI 0.14-3.51), or clinicopathological type (OR $1.14,95 \%$ CI $0.69-1.86)$. Moreover, a high ALDH1 expression was significantly associated with overall survival (HR 1.56, 95\% CI 1.21-2.02) but not with disease-free survival (HR $1.38,95 \%$ CI $0.99-1.93)$.

Conclusion: The meta-analysis indicates that increasing ALDH1 predicts poor prognosis and clinicopathological characteristics in OC. Future studies are needed to explore tailored treatments that directly target ALDH1 for the improvement of survival in OC.

Keywords: ovarian cancer, ALDH1, prognosis, clinicopathological characteristics, metaanalysis

\section{Introduction}

Ovarian cancer (OC) is the most common lethal gynecological malignancy, followed by cervical cancer and endometrial cancer, and has the third highest morbidity rate of gynecological malignancies. Based on its mortality rate, OC is first among all gynecologic malignancies. ${ }^{1} \mathrm{OC}$ is characterized by its insidious onset, aggressiveness, rapid metastasis, and poor prognosis. Furthermore, given the lack of specific symptoms and methods for early screening, many patients with epithelial ovarian cancer (EOC) are diagnosed at an advanced stage, and their 5-year survival rate is only $25 \%-30 \% .^{2}$ In the clinic, EOC is the most common histological subtype of OC, which also includes serous OC, clear cell OC, endometrioid adenocarcinoma, and mixed cell OC. Several independent prognostic factors such as 
disease stage, age, metastasis, classification, and residual tumor bulk have been identified in previous investigations.

Cancer stem cells (CSCs) are defined as a small population of cancer cells that have high tumorigenicity. Furthermore, the CSC hypothesis has been formulated to explain tumor occurrence and recurrence. ${ }^{3}$ Increasing evidence has indicated that $\mathrm{CSC}$ s have self-renewal capacity; ${ }^{4}$ therefore, CSCs are thought to be responsible for tumor progression, metastasis, and therapeutic resistance.

Aldehyde dehydrogenase 1 (ALDH1), an important marker of stem-like cells in many malignant tumors, ${ }^{5,6}$ is a zinc-containing enzyme with oxidation and detoxification functions. $^{7}$ To this day, the correlation between ALDH1 expression and $\mathrm{OC}$ has been widely studied, but the results remain controversial. Several studies have suggested that the high expression of ALDH1 is related to disease stage, age, metastasis, classification, and residual tumor bulk, but other studies did not reach these conclusions. ${ }^{8,9}$ We thus conducted a meta-analysis to reveal the prognostic significance of ALDH1 for overall survival (OS) and disease-free survival (DFS) as well as the association between ALDH1 and clinicopathological features in patients with OC.

\section{Materials and methods}

\section{Literature search}

Several electronic databases, including PubMed, Embase, and China Biology Medicine (CBM), were independently searched by two researchers (W Zhao and C Zang) through October, 2017. We used the MeSH/Emtree terms, combining free text words that were properly adjusted for the different databases in all of the search strategies. The following keywords were used for searching: (“ALDH1" OR "aldehyde dehydrogenase 1" OR “ALDH1 enzyme") AND ("ovarian cancer" OR “ovarian tumor" OR “ovarian carcinoma” OR "ovarian neoplasm”). For a detailed search strategy please refer to the Supplementary materials.

\section{Inclusion and exclusion criteria}

All of the studies in this meta-analysis had to meet the following inclusion criteria: 1) patient diagnoses were confirmed as OC by pathology, 2) the outcome of interest was the clinicopathological and/or prognostic relationship between ALDH1 and OC, 3) immunohistochemistry (IHC) detection methods were used to detect specific ALDH1 antigens with monoclonal anti-human ALDH1, and 4) the outcome measures of interest could be extracted directly or calculated from the published data indirectly. The exclusion criteria were as follows: 1) studies that were reviews, letters or conference papers, 2) studies that were not performed in humans, and 3) papers that failed to report sufficient data.

\section{Data extraction}

Extraction of data was independently performed by two reviewers (W Zhao and C Zang) in accordance with a predesigned and standardized form, and discrepancies were settled through a consensus discussion. The following information was extracted from the included studies: first author, year, patient source, type of patients, median/mean age, sample size, technique, cutoff value, median follow-up, OS/DFS, and clinicopathological features. For the specific data extracted, see refer to the Supplementary materials.

\section{Quality assessment}

The quality of the included cohort studies was evaluated according to the Newcastle-Ottawa Scale (NOS). ${ }^{10}$ Two reviewers (W Zhao and C Zang) evaluated all of the studies independently, and discrepancies were solved by consensus. The standards included three aspects: 1) selection of the research groups, 2) comparability of the groups, and 3) measurement of exposure factors or outcome. Scores ranged from 0 to 9 , and a score $\geq 6$ indicated a high quality.

\section{Statistical analysis}

Data for the clinicopathological features were extracted from available studies of odds ratios (ORs). The hazard ratios (HRs) and 95\% CIs were used to summarize survival outcomes. We would have obtained pooled HRs and 95\% CIs provided the statistical data were reported in the study. When HRs and 95\% CIs were not directly reported in the studies, survival information was extracted from the Kaplan-Meier curves using Engauge Digitizer 4.1 (http://digitizer.sourceforge.net/) and used to estimate HR. ${ }^{11}$ Statistical heterogeneity was assessed using both a chi-square-based $Q$-test and $I^{2}$ statistic, which describe the percentage of total variation across studies caused by heterogeneity rather than by chance. Studies with an $I^{2}>50 \%$ or $P<0.1$ were considered to have significant heterogeneity, and a random-effects model test was conducted. Otherwise, the fixed-effects model test was adopted. Funnel plots and Egger's test were used to evaluate publication bias. Sensitivity analysis was carried out to evaluate the effect of a single study on all the results and to find the origin of heterogeneity. $P<0.05$ was considered to be statistically significant. All the calculations were performed by Stata 13.0 (StataCorp LP, College Station, TX, USA). 


\section{Results}

\section{Literature search}

There were 165 records found from database searches according to our search strategy, of which 45 papers were duplicates. Through screening title and abstract, 24 irrelevant papers were excluded; then 79 papers, including 13 reviews, 33 experimental studies, 28 papers lacking of data, 3 papers without immunohistochemistry (IHC) of ALDH1 detection and 2 papers unable to be obtained, were excluded after reading the full text. Finally, 17 eligible papers (18 studies) involving 2,531 patients were included in our meta-analysis., ${ }^{5,8,9,12-25}$ The detailed literature search and paper selection are shown in Figure 1.

\section{Characteristics of the included papers}

The details of the 17 included manuscripts, including 2,531 patients (sample size median: 88 [37-442]), selected from the literature search are summarized in Table 1. In total, 13 eligible papers (14 studies) with 1,841 patients were analyzed for clinicopathological features, and 15 qualified studies with 2,379 patients were analyzed for survival outcomes. The studies were conducted in seven countries (China, Finland, Korea, Japan, Germany, Norway, and the USA) and were published between 2009 and 2017. All included studies detected ALDH1 using IHC. Among included papers, ALDH1 expression in tumor cells was most commonly

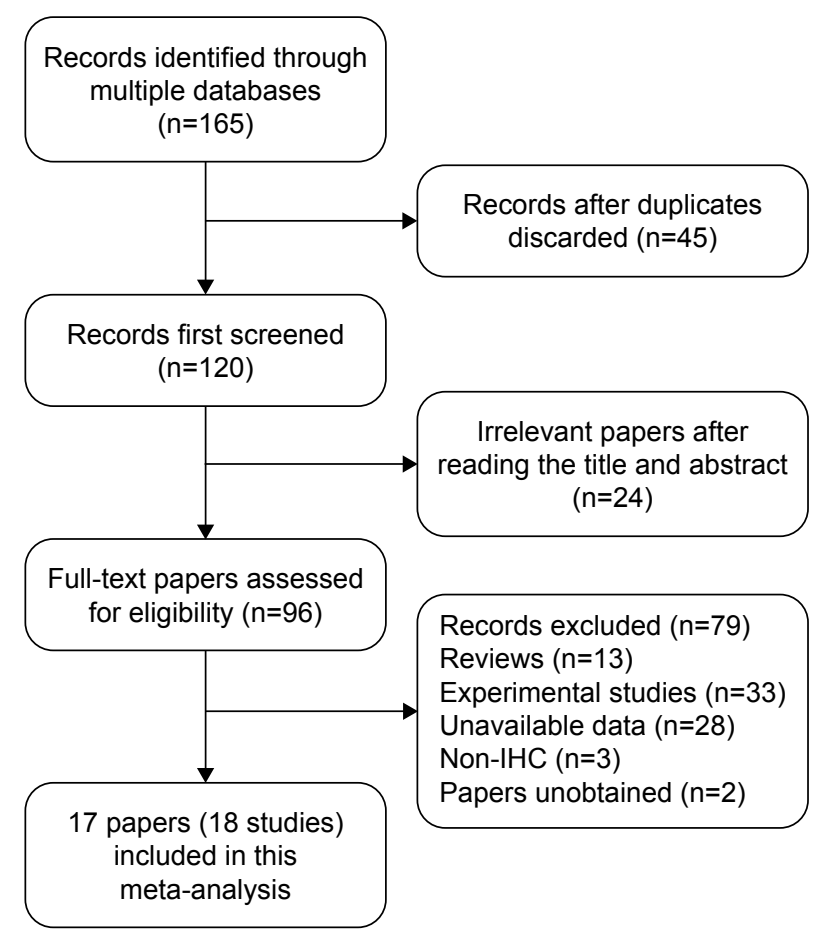

Figure I Flow diagram of the studies identified, included, and excluded. Abbreviation: $\mathrm{IHC}$, immunohistochemistry. evaluated based on the percentage of positively stained cells or the semi-quantitative immunoreactivity score.

\section{Quality assessment}

Each of the 18 included studies was evaluated using the NOS, as described previously. Scores are shown in Table 2, in which " $\star$ " represents 1 point, " $X$ ” represents 0 point, and "-_" represents uncertain points. Deng's quality evaluation was not carried out due to lack of detailed information on the exposed group and the nonexposed group. Except for studies of Xue et al and Jing et al, the others are of high quality. Owing to lack of follow-up, the studies of Xue et al and Jing et al only have 5 points. Research of Kuroda et al and Wang et al could not score because follow-up occurred before the study, nor could studies of Chang et al and Huang et al, because they failed to control comparisons among groups. The studies of Ayub et al, Liebscher et al, and Landen et al did not describe the inter-group comparability. However, studies of Kuroda et al and Ruscito et al strictly controlled the inter-group comparability. The remaining studies only had one point in inter-group comparability. The differences between the inter-groups may primarily stem from age, tumor stage, grade, or lymph node metastasis.

\section{Effect of ALDHI expression on clinicopathological parameters}

Age, tumor size, tumor location, ascite status, resistance status, and clinicopathological type

We assessed the association between age, tumor size, tumor location, ascite status, resistance status, clinicopathological type, and ALDH1 expression. As illustrated in Table 3, ALDH1 expression was not associated with age ( $<55$ vs $\geq 55$ : OR $0.90,95 \%$ CI $0.25-3.28, I^{2} 74.1 \%$ ), tumor size $(<8 \mathrm{~cm}$ vs $\geq 8 \mathrm{~cm}$ : OR $1.13,95 \%$ CI $0.75-1.71$, $I^{2} 0.0 \%$ ), tumor location (unilateral vs bilateral: OR 0.69 , 95\% CI $0.22-2.13, I^{2} 71.5 \%$ ), ascite status (yes vs no: OR $0.74,95 \%$ CI $0.49-1.11, I^{2} 0.0 \%$ ), resistance status (yes vs no: OR $0.70,95 \%$ CI $0.14-3.51, I^{2} 87.6 \%$ ), or clinicopathological type (S vs C: OR 1.14, 95\% CI 0.69 $1.86, I^{2} 0.0 \%$ ). The results are shown in Figure $2 \mathrm{~A}-\mathrm{F}$ and Table 3.

\section{International Federation of Gynecology and Obstetrics (FIGO) stage}

The incidence of ALDH1 in FIGO stage may indicate the degree of FIGO stage (III-IV vs I-II: OR 2.02, 95\% CI 1.16-3.52, $\left.I^{2} 78.0 \%\right)$. Subgroup analyses were adopted on sample size $(<100$ : OR $3.34,95 \%$ CI $2.20-5.08$, 


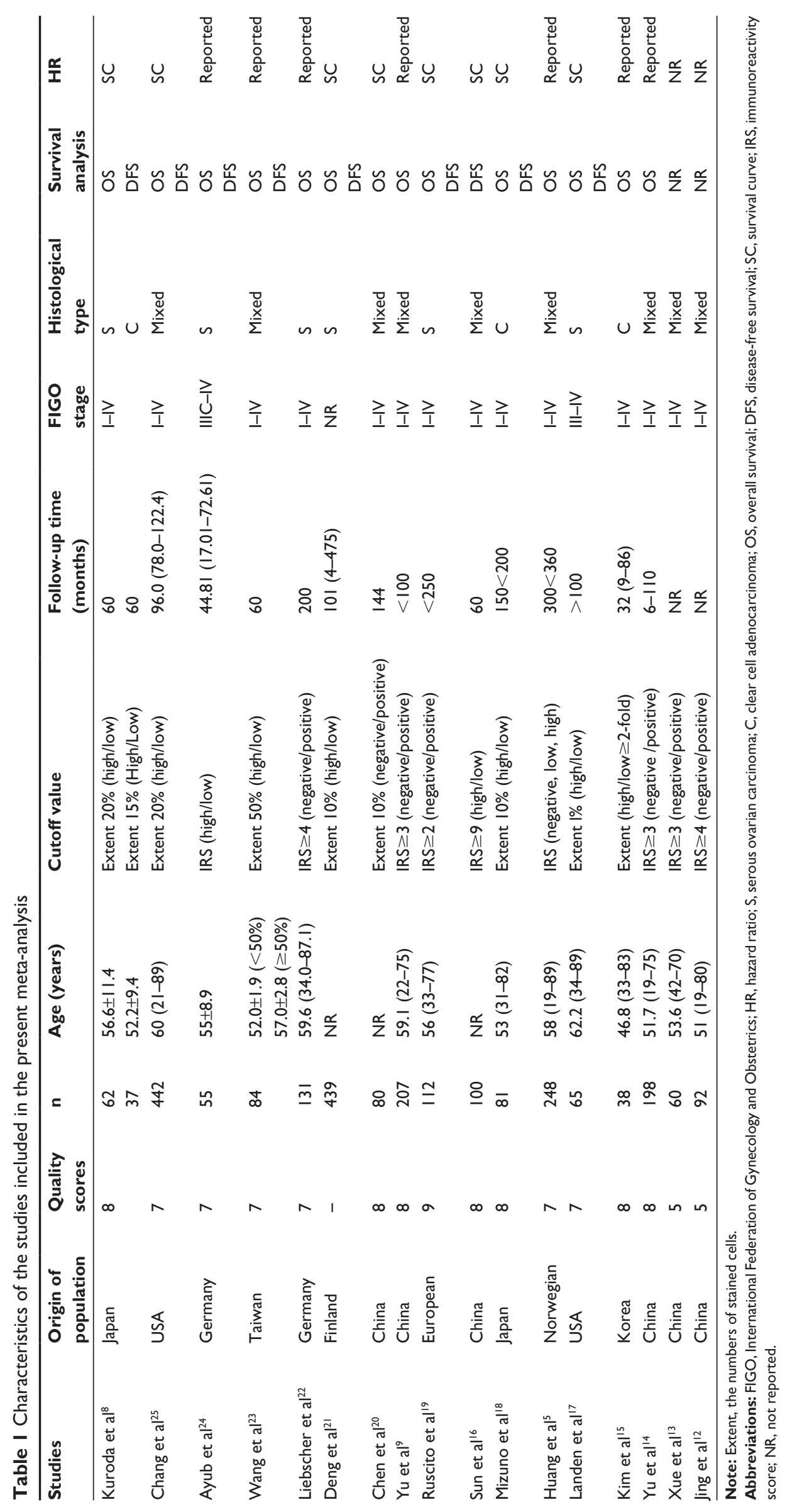


Table 2 Quality scores of included studies using NOS

\begin{tabular}{|c|c|c|c|c|c|c|c|c|c|}
\hline Studies & $\begin{array}{l}\text { Representativeness } \\
\text { of exposed group }\end{array}$ & $\begin{array}{l}\text { Representativeness } \\
\text { of non-exposed } \\
\text { group }\end{array}$ & $\begin{array}{l}\text { Sources of } \\
\text { exposure } \\
\text { factor }\end{array}$ & $\begin{array}{l}\text { Not } \\
\text { observed } \\
\text { outcome } \\
\text { at first }\end{array}$ & Comparability & $\begin{array}{l}\text { Evaluation } \\
\text { of outcome }\end{array}$ & $\begin{array}{l}\text { Enough } \\
\text { follow- } \\
\text { up time }\end{array}$ & $\begin{array}{l}\text { Adequacy } \\
\text { of follow- } \\
\text { up }\end{array}$ & Scores \\
\hline $\begin{array}{l}\text { Kuroda } \\
\text { et al }\end{array}$ & $\star$ & $\star$ & $\star$ & $x$ & $\star \star$ & $\star$ & $\star$ & $\star$ & 8 \\
\hline Chang et $\mathrm{al}^{25}$ & $\star$ & $\star$ & $\star$ & $\star$ & $\times$ & $\star$ & $\star$ & $\star$ & 7 \\
\hline Ayub et $\mathrm{al}^{24}$ & $\star$ & $\star$ & $\star$ & $\star$ & - & $\star$ & $\star$ & $\star$ & 7 \\
\hline Wang et $\mathrm{a}^{23}$ & $\star$ & $\star$ & $\star$ & $x$ & $\star$ & $\star$ & $\star$ & $\star$ & 7 \\
\hline $\begin{array}{l}\text { Liebscher } \\
\text { et } \mathrm{a}^{22}\end{array}$ & $\star$ & $\star$ & $\star$ & $\star$ & - & $\star$ & $\star$ & $\star$ & 7 \\
\hline Deng et $\mathrm{a}^{21}$ & The quality evaluation & cannot be applied witho & ut detailed infe & formation & & & & & \\
\hline Chen et $\mathrm{a}^{20}$ & $\star \quad 7 \quad 1-$ & $\star$ & $\star$ & $\star$ & $\star$ & $\star$ & $\star$ & $\star$ & 8 \\
\hline Yu et al ${ }^{9}$ & $\star$ & $\star$ & $\star$ & $\star$ & $\star$ & $\star$ & $\star$ & $\star$ & 8 \\
\hline $\begin{array}{l}\text { Ruscito } \\
\text { et al }{ }^{19}\end{array}$ & $\star$ & $\star$ & $\star$ & $\star$ & $\star \star$ & $\star$ & $\star$ & $\star$ & 9 \\
\hline $\begin{array}{l}\text { Mizuno } \\
\text { et al }{ }^{18}\end{array}$ & $\star$ & $\star$ & $\star$ & $\star$ & $\star$ & $\star$ & $\star$ & $\star$ & 8 \\
\hline Huang et $\mathrm{al}^{5}$ & $\star$ & $\star$ & $\star$ & $\star$ & $x$ & $\star$ & $\star$ & $\star$ & 7 \\
\hline $\begin{array}{l}\text { Landen } \\
\text { et al }{ }^{17}\end{array}$ & $\star$ & $\star$ & $\star$ & $\star$ & - & $\star$ & $\star$ & $\star$ & 7 \\
\hline Sun et $\mathrm{al}^{16}$ & $\star$ & $\star$ & $\star$ & $\star$ & $\star$ & $\star$ & $\star$ & $\star$ & 8 \\
\hline Kim et $\mathrm{al}^{15}$ & $\star$ & $\star$ & $\star$ & $\star$ & $\star$ & $\star$ & $\star$ & $\star$ & 8 \\
\hline Yu et al ${ }^{14}$ & $\star$ & $\star$ & $\star$ & $\star$ & $\star$ & $\star$ & $\star$ & $\star$ & 8 \\
\hline Xue et $\mathrm{al}^{13}$ & $\star$ & $\star$ & $\star$ & $\times$ & $\star$ & $\star$ & $x$ & $x$ & 5 \\
\hline Jing et $\mathrm{al}^{12}$ & $\star$ & $\star$ & $\star$ & $\times$ & $\star$ & $\star$ & $x$ & $x$ & 5 \\
\hline
\end{tabular}

Notes: “ ” represents I point, “ $\times$ ” represents 0 point, and “-” represents uncertain points.

Abbreviation: NOS, Newcastle-Ottawa Scale.

$I^{2} 40.1 \%$; $\geq 100$ : OR $1.18,95 \%$ CI $\left.0.53-2.60, I^{2} 85.1 \%\right)$ and ethnicity (Asian: OR 2.82, 95\% CI 2.13-3.72, $I^{2} 23.1 \%$; nonAsian: OR 0.45, 95\% CI 0.29-0.71, $I^{2} 0.0 \%$ ). The results are shown in Figure $3 \mathrm{~A}$ and Table 3.

\section{Histopathologic grade}

Pooled OR from eight studies is shown in Figure 3B and Table 3, indicating that high ALDH1 expression was not associated with the degree of histopathologic grade (G1 vs
G2+G3: OR 0.53, 95\% CI 0.23-1.27, $I^{2} 83.2 \%$ ). Subgroup analyses were only adopted on sample size $(<100$ : OR $0.29,95 \%$ CI $0.06-1.38, I^{2} 81.9 \%$; $\geq 100$ : OR $0.87,95 \%$ CI $0.30-2.54, I^{2} 85.2 \%$ ) due to the limited number of studies on ethnicity.

\section{Lymph node metastasis}

The pooled OR from eight studies is shown in Figure 3C and Table 3, indicating that a high ALDH1 expression was

Table 3 Effects of ALDHI expression on clinicopathological parameters and survival outcome

\begin{tabular}{|c|c|c|c|c|c|c|c|c|c|}
\hline \multirow[t]{2}{*}{ Clinicopathological variables } & \multirow[t]{2}{*}{$\begin{array}{l}\text { No of } \\
\text { studies }\end{array}$} & \multirow[t]{2}{*}{ Cases } & \multirow[t]{2}{*}{$\begin{array}{l}\text { Selected } \\
\text { model }\end{array}$} & \multicolumn{2}{|c|}{$\begin{array}{l}\text { Heterogeneity } \\
\text { test }\end{array}$} & \multirow[t]{2}{*}{$\begin{array}{l}\text { OR/HR } \\
(95 \% \mathrm{CI})\end{array}$} & \multirow[t]{2}{*}{$\mathbf{Z}$} & \multirow[t]{2}{*}{$\mathbf{P 2}$} & \multirow[t]{2}{*}{$\boldsymbol{P}_{\text {Begg }}$} \\
\hline & & & & $I^{2}(\%)$ & $\mathbf{P I}$ & & & & \\
\hline Age ( $>50$ years $/<50$ years) & 2 & 329 & Random & 74.1 & 0.049 & $0.90(0.25-3.28)$ & 0.16 & 0.870 & - \\
\hline Tumor size $(\geq 8 \mathrm{~cm} /<8 \mathrm{~cm})$ & 2 & 405 & Fixed & 0.0 & 0.900 & I.I3 (0.75-I.7I) & 0.58 & 0.562 & - \\
\hline Tumor grade (GI/G2+G3) & 8 & $\mathrm{I}, 067$ & Random & 83.2 & 0.000 & $0.53(0.23-1.27)$ & 1.42 & 0.156 & 0.386 \\
\hline Tumor stage (III+IV/I-II) & 14 & $|, 84|$ & Random & 78.0 & 0.000 & $2.02(1.16-3.52)$ & 2.50 & 0.012 & 0.743 \\
\hline LNM (yes/no) & 8 & $\mathrm{I}, 176$ & Random & 67.9 & 0.003 & $1.91(1.01-3.61)$ & 1.98 & 0.048 & 1.000 \\
\hline Ascite (yes/no) & 2 & 405 & Fixed & 0.0 & 0.869 & $0.74(0.49-1.1 \mathrm{I})$ & 1.45 & 0.147 & - \\
\hline Chemoresponse (sensitive/resistant) & 3 & 592 & Random & 87.6 & 0.000 & $0.70(0.14-3.5 I)$ & 0.44 & 0.664 & - \\
\hline Tumor location (unilateral/bilateral) & 2 & 287 & Random & 71.5 & 0.061 & $0.69(0.22-2.13)$ & 0.65 & 0.517 & - \\
\hline Distant metastasis (yes/no) & 2 & 245 & Random & 58.6 & 0.120 & $5.43(1.44-20.42)$ & 2.50 & 0.012 & - \\
\hline Clinicopathological type (S/C) & 8 & $\mathrm{I}, 450$ & Random & 0.0 & 0.977 & $1.14(0.69-1.86)$ & 0.50 & 0.614 & 0.711 \\
\hline OS & 15 & 2,279 & Random & 74.0 & 0.000 & $1.56(1.21-2.02)$ & 3.39 & 0.001 & 0.083 \\
\hline DFS & 10 & I,477 & Random & 76.1 & 0.000 & $1.38(0.99-1.93)$ & 1.92 & 0.055 & 0.788 \\
\hline
\end{tabular}

Abbreviations: ALDHI, aldehyde dehydrogenase I; OR, odds ratio; HR, hazard ratio; Cl, confidence interval; LNM, lymph node metastasis; S, serous ovarian carcinoma; C, clear cell adenocarcinoma; OS, overall survival; DFS, disease-free survival. 


\section{A}

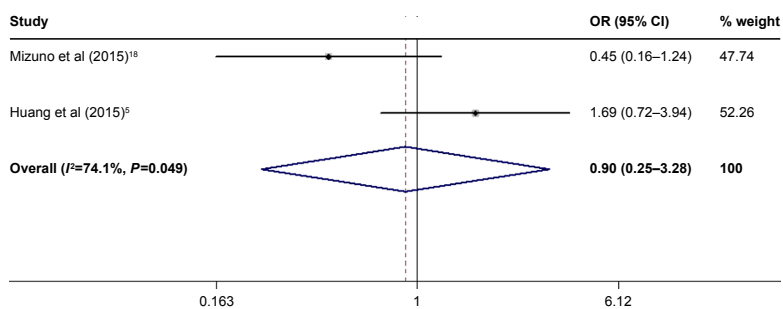

C

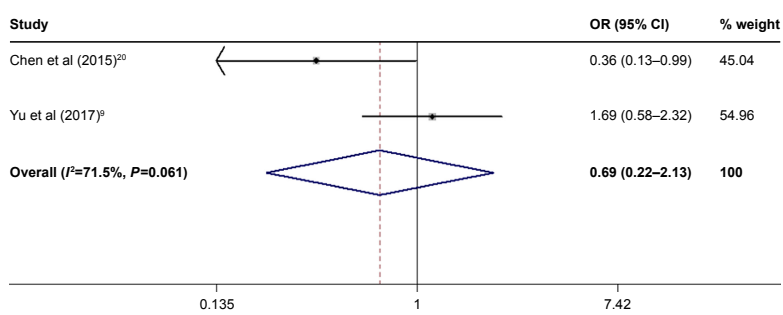

E study

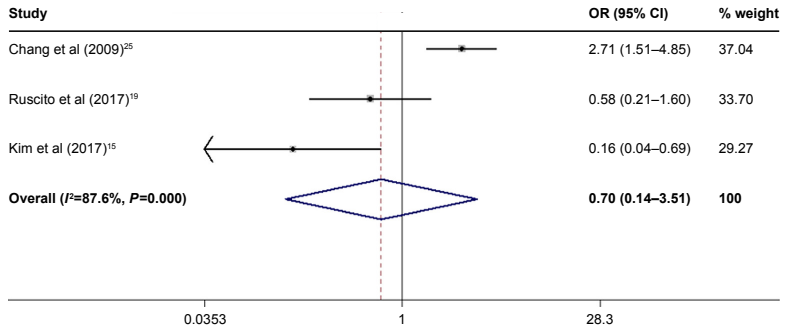

B

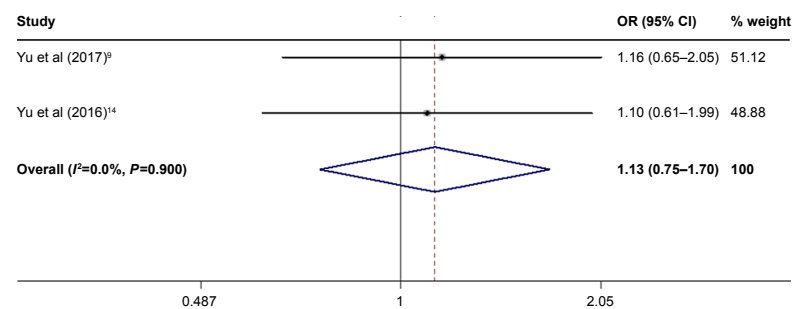

D

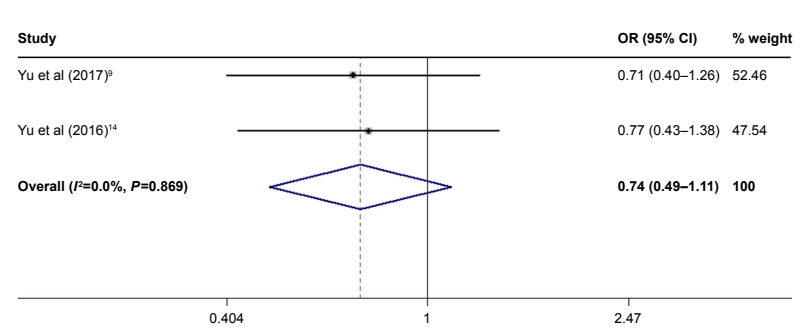

F study

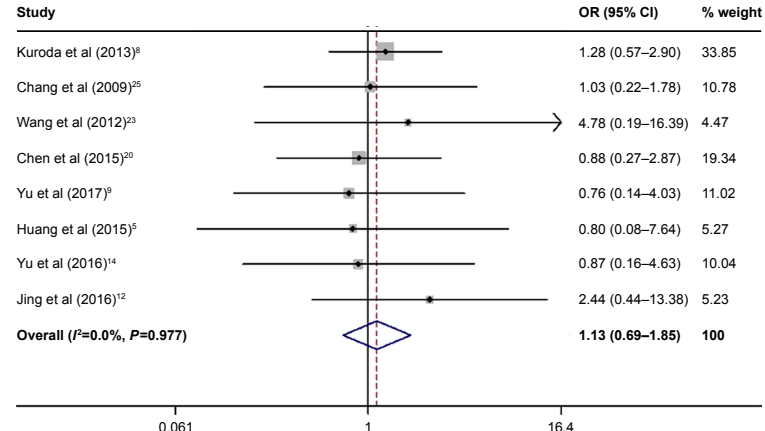

Figure 2 Forest plot of ORs for the association between ALDHI expression and age, tumor size, tumor location, ascite status, resistance status, and clinicopathological type.

Notes: (A) Age ( $<55$ vs $\geq 55$ years), (B) tumor size ( $<8 \mathrm{~cm}$ vs $\geq 8 \mathrm{~cm}$ ), (C) tumor location (unilateral vs bilateral), (D) ascite status (yes vs no), (E) resistance status (yes vs no), and (F) clinicopathological type ( $\mathrm{S}$ vs $\mathrm{C})$. Weights are from random-effects analysis.

Abbreviations: ALDHI, aldehyde dehydrogenase I; OR, odds ratio; S, serous ovarian carcinoma; C, clear cell adenocarcinoma.

significantly associated with lymph node status (yes vs no: OR 1.91, 95\% CI 1.01-3.61, $\left.I^{2} 67.9 \%\right)$. Subgroup analyses were adopted on sample size $(<100$ : OR $2.42,95 \%$ CI $0.82-7.11, I^{2} 67.6 \%$; $\geq 100$ : OR 1.91, 95\% CI 1.00-3.60, $\left.I^{2} 75.0 \%\right)$. All patients on lymph node metastasis were from Asia.

\section{Distant metastasis}

The ORs for distant metastasis were only available in two studies, indicating that high ALDH1 expression was significantly associated with distant metastasis (yes vs no: OR 5.43, 95\% CI 1.44-20.42, $\left.I^{2} 58.6 \%\right)$. The results are shown in Figure 3D and Table 3.

\section{Effect of ALDHI expression on survival outcome}

We assessed the association between increasing ALDH1 levels and survival outcomes of OC patients. In total, 10 eligible studies with 1,477 patients were analyzed for
DFS, and 15 qualified studies with 2,279 patients were analyzed for OS. The results are shown in Figure 3E and F and Table 3, indicating that a high ALDH1 expression was significantly associated with OS (high $[\mathrm{H}]$ vs low [L]: HR $1.56,95 \%$ CI 1.21-2.02, $I^{2} 74.0 \%$ ) but not with DFS (H vs L: HR 1.38, 95\% CI 0.99-1.93, $\left.I^{2} 76.1 \%\right)$. Subgroup analyses for OS were adopted on sample size (<100: HR 2.37, 95\% CI 1.79-3.14, $I^{2} 0.0 \%$; $\geq 100$ : HR $1.16,95 \%$ CI $0.92-1.46$, $I^{2} 68.5 \%$ ) and ethnicity (Asian: HR 2.07, 95\% CI 1.64-2.63, $I^{2} 0.0 \%$; non-Asian: HR 1.18, 95\% CI 0.90-1.54, $I^{2} 72.1 \%$ ). The subgroup analyses for DFS were performed on sample size (<100: HR 1.50, 95\% CI 1.11-2.03, $I^{2} 46.3 \%$; $\geq 100$ : HR 1.26, 95\% CI 0.78-2.04, $I^{2} 87.2 \%$ ) and ethnicity (Asian: HR 1.45, 95\% CI 1.07-2.01, $I^{2} 55.2 \%$; non-Asian: HR 1.34, 95\% CI 0.86-2.07, $\left.I^{2} 84.0 \%\right)$.

\section{Publication bias}

Owing to the limited number of OC studies, we did not assess publication bias using Begg's test, Egger's test or 
$A_{\text {study }}$

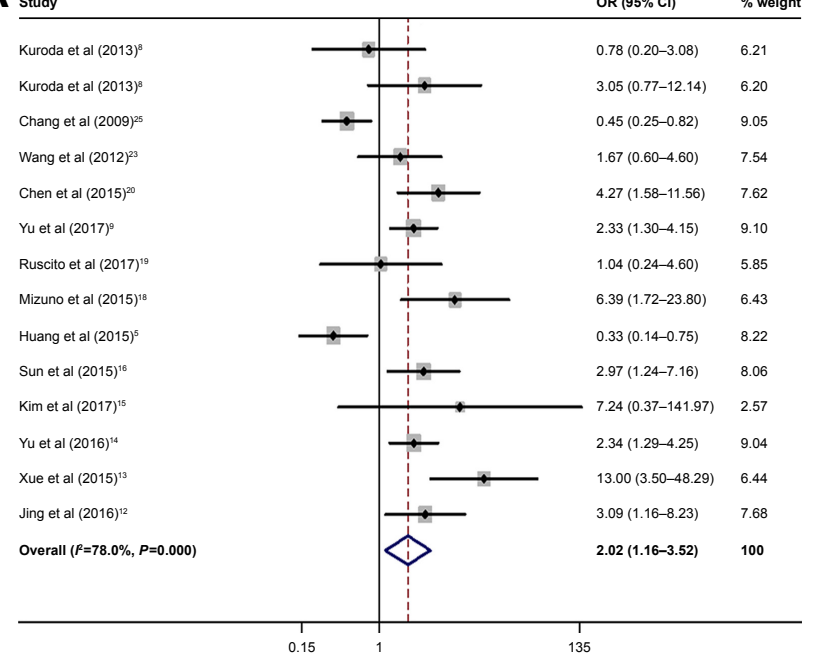

$\mathbf{C}_{\text {suat }}$

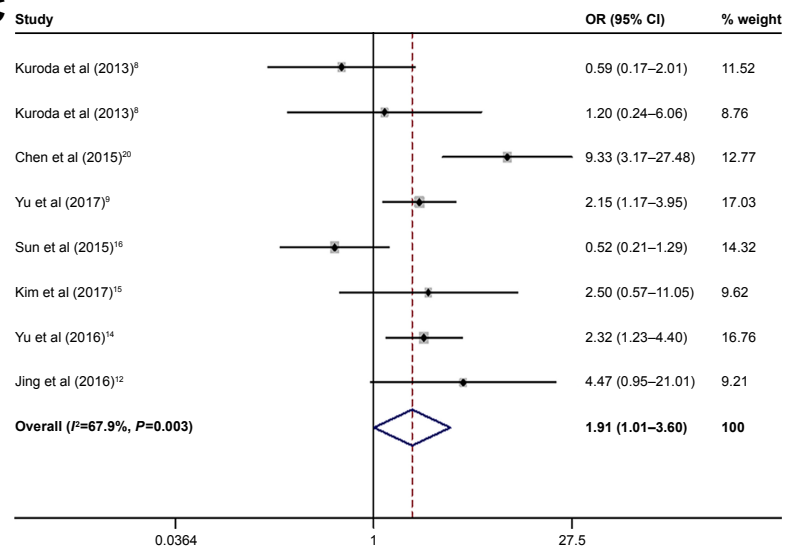

$E_{\text {suat }}$

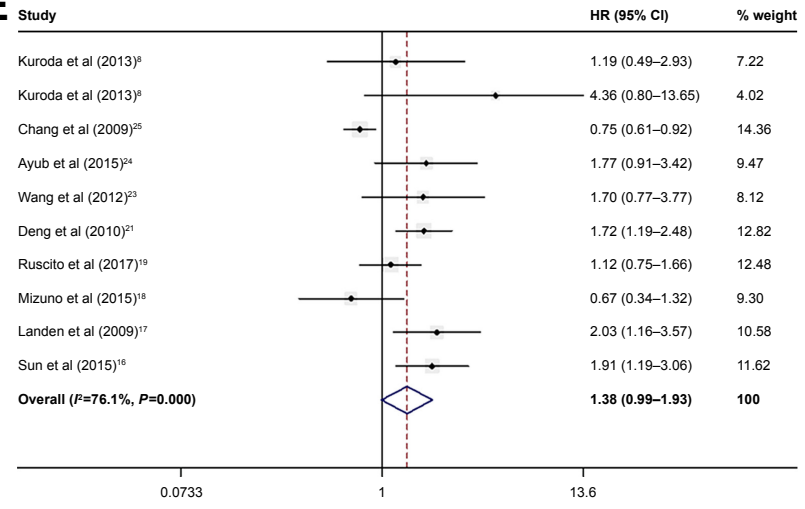

$\mathbf{B}_{\text {study }}$

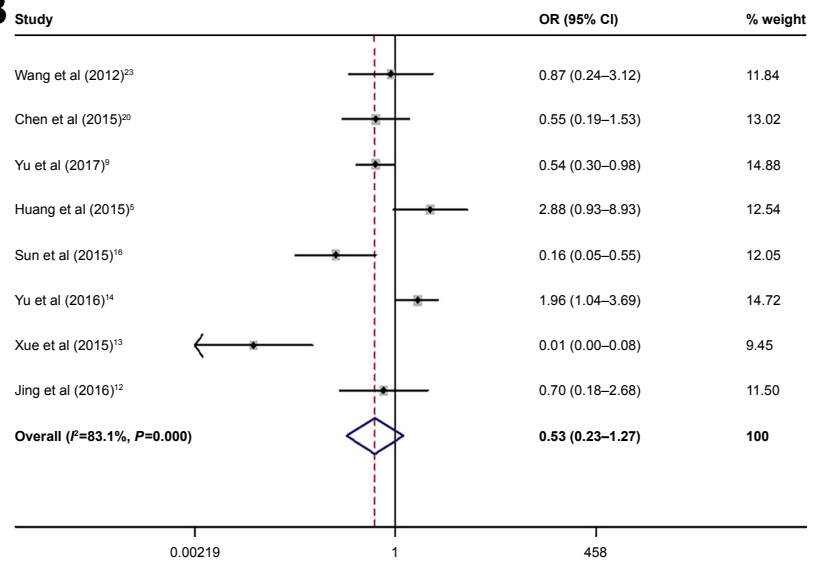

$\mathbf{D}_{\text {study }}$

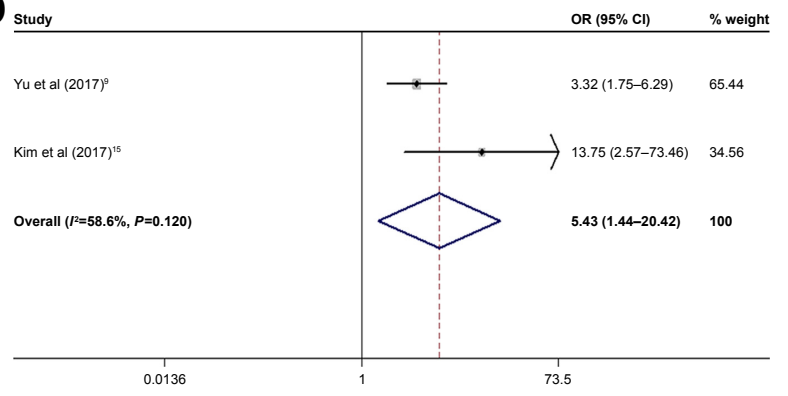

$\mathbf{F}_{\text {study }}$

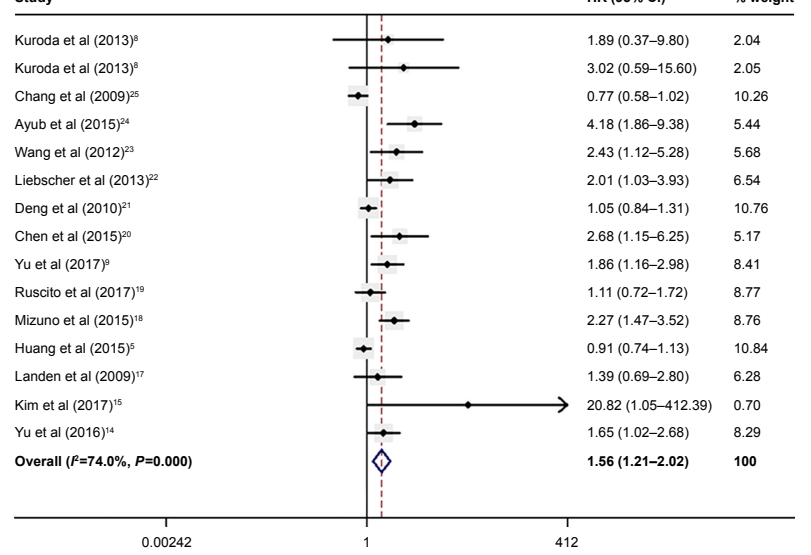

Figure 3 Forest plot of ORs/HRs for the association between ALDHI expression and FIGO stage, histopathologic grade, lymph node metastasis, distant metastasis, DFS, and OS.

Notes: (A) FIGO stage (III-IV vs I-II), (B) histopathologic grade (GI vs G2+G3), (C) lymph node metastasis (yes vs no), (D) distant metastasis (yes vs no), (E) DFS (H vs L), and $(\mathbf{F})$ OS (H vs $L)$. Weights are from random-effects analysis.

Abbreviations: ALDHI, aldehyde dehydrogenase I; OR, odds ratio; HR, hazard ratio; FIGO, International Federation of Gynecology and Obstetrics; DFS, disease-free survival; OS, overall survival; $\mathrm{H}$, high; L, low.

funnel plots for age, tumor size, tumor location, ascite status, and resistance. The funnel plots appeared to be symmetric, showing no evidence of publication bias for the other pooled clinicopathological characteristics and survival outcomes.
In addition, both tests also indicated that there was no publication bias for pooled tumor grade $\left(P_{\mathrm{Begg}}=0.231\right)$, FIGO stage $\left(P_{\text {Begg }}=0.242\right)$, lymph node metastasis $\left(P_{\text {Begg }}=0.950\right)$, or clinicopathological type $\left(P_{\text {Begg }}=0.868\right)$ as well as DFS 
$\left(P_{\text {Begg }}=0.788\right)$ or OS $\left(P_{\text {Begg }}=0.083\right)$. The results are shown in Figure $4 \mathrm{~A}-\mathrm{F}$ and Table 3.

\section{Sensitivity analysis}

We performed a sensitivity analysis to assess the stability of our results regarding clinicopathological characteristics, DFS, and OS in OC patients, and results are shown in
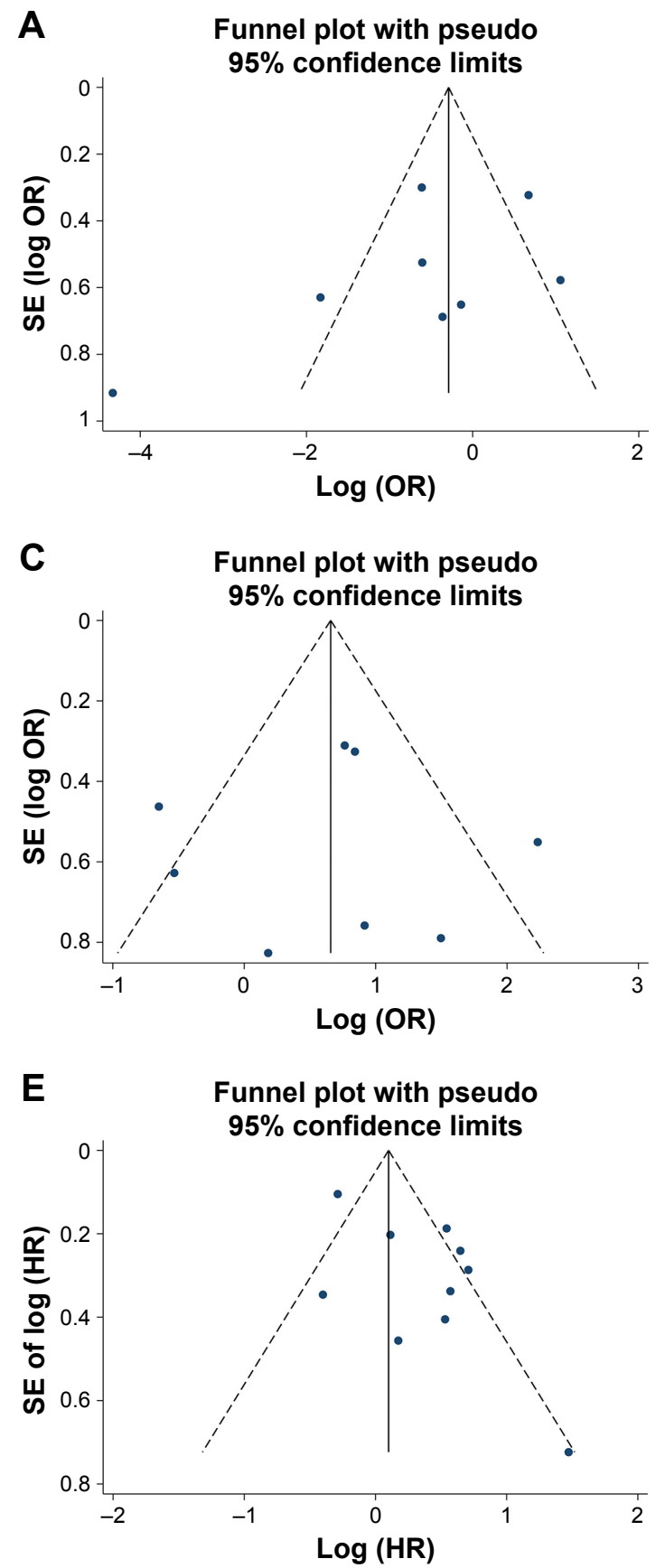

Figure 5A-F. No significant changes were identified in the pooled results when a study was removed, indicating the results of this meta-analysis are robust.

\section{Discussion}

In recent years, in spite of improvement in diagnosis and treatment, $\mathrm{OC}$ is still characterized by late clinical
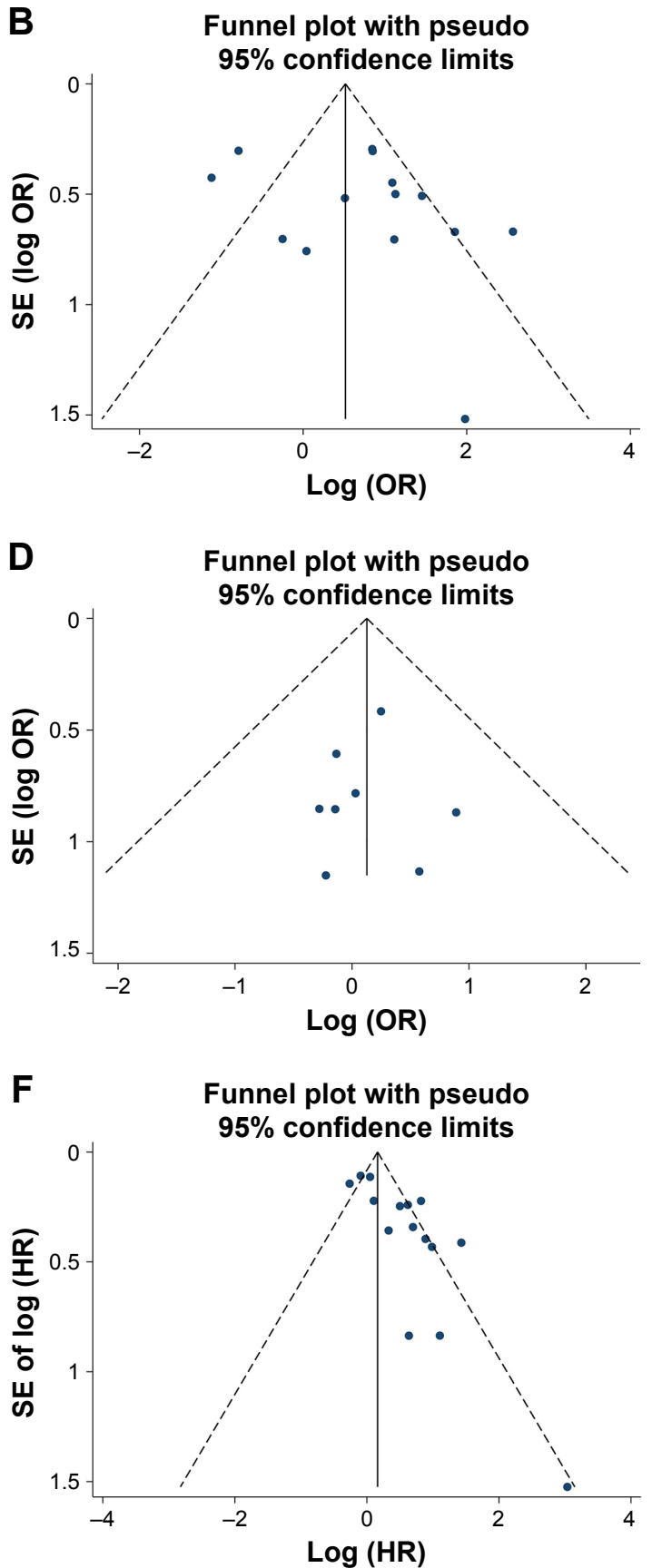

Figure 4 Funnel plot for all the eligible studies that provided ORs/HRs for high ALDHI expression with respect to histopathologic grade, FIGO stage, lymph node metastasis, clinicopathological type, DFS, and OS.

Notes: (A) Histopathologic grade (GI vs G2+G3), (B) FIGO stage (III-IV vs I-II), (C) lymph node metastasis (yes vs no), (D) clinicopathological type (S vs C), (E) DFS ( $\mathrm{H}$ vs $\mathrm{L})$, and (F) OS ( $\mathrm{H}$ vs $\mathrm{L})$.

Abbreviations: ALDHI, aldehyde dehydrogenase I; OR, odds ratio; HR, hazard ratio; FIGO, International Federation of Gynecology and Obstetrics; DFS, disease-free survival; OS, overall survival; S, serous ovarian carcinoma; C, clear cell adenocarcinoma; H, high; L, low; SE, standard error; log, Napierian logarithm. 
A

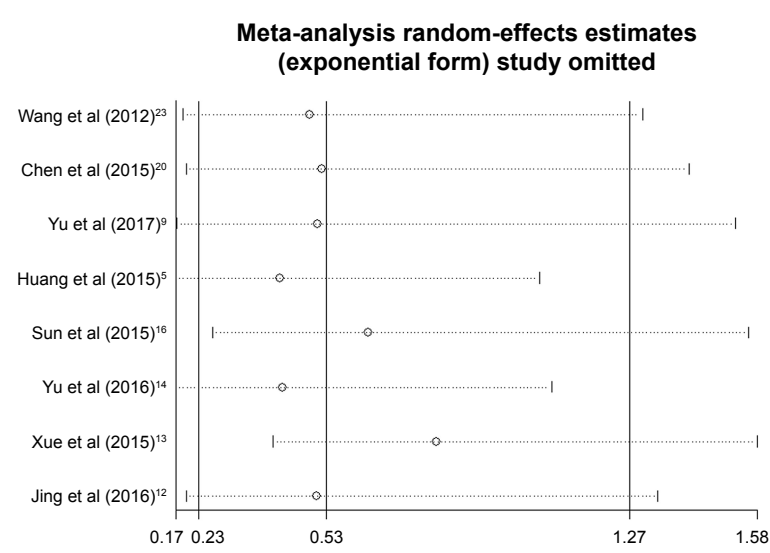

C

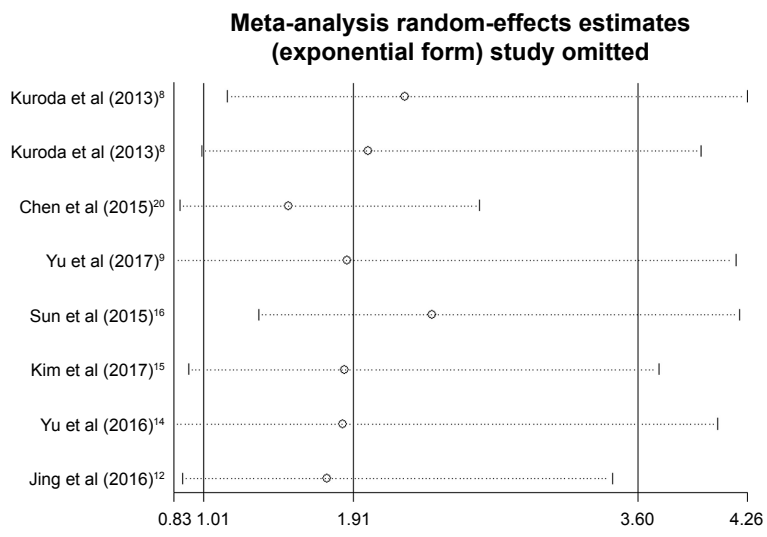

E

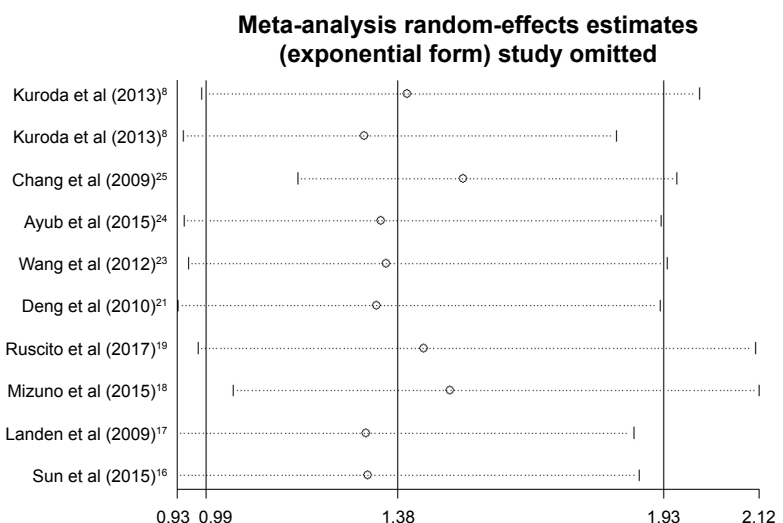

B

Meta-analysis random-effects estimates (exponential form) study omitted

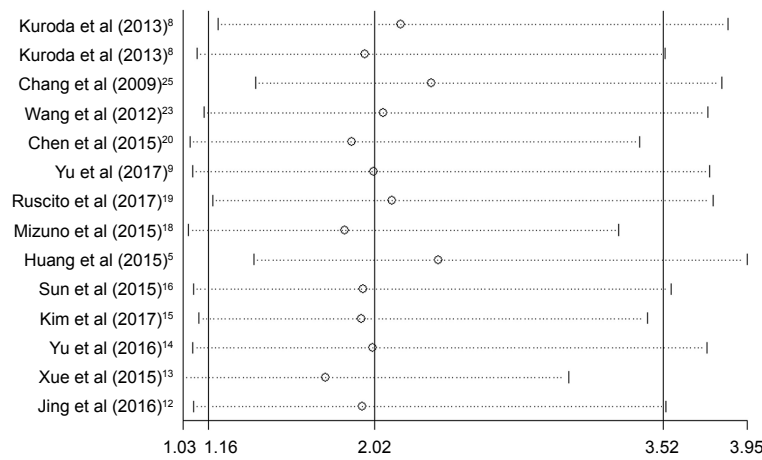

D
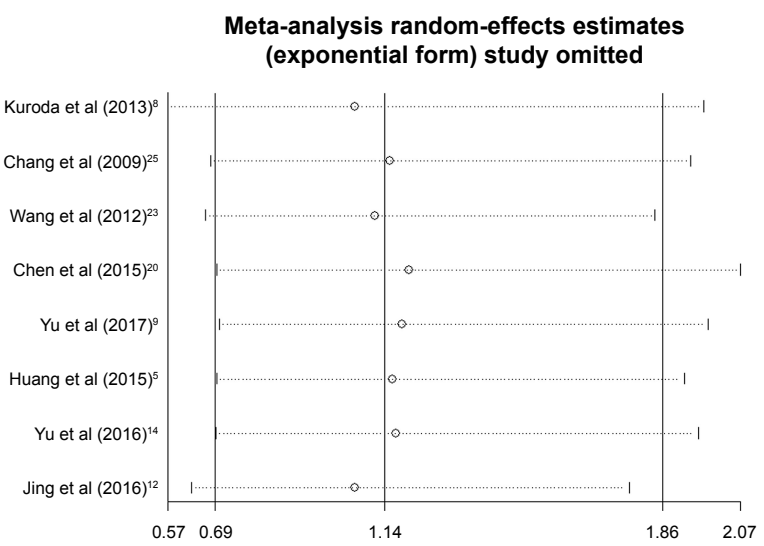

$\mathbf{F}$

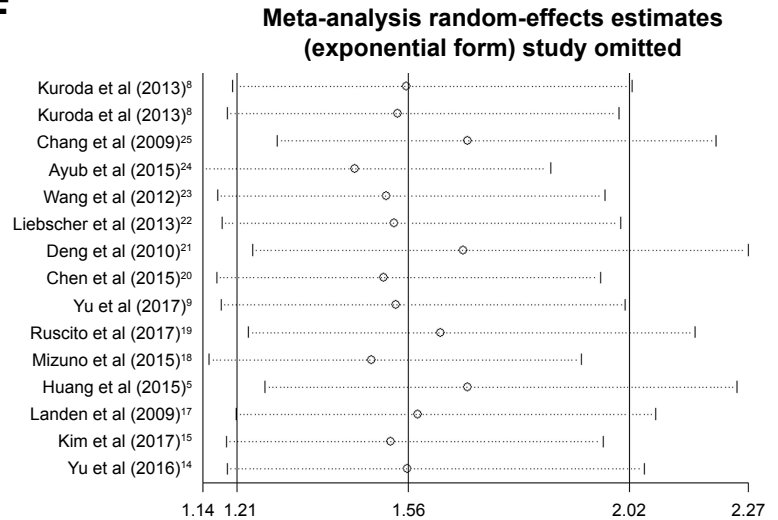

Figure 5 Sensitivity analysis for all the eligible studies that provided ORs/HRs of high ALDHI expression for histopathologic grade, FIGO stage, lymph node metastasis, clinicopathological type, DFS, and OS.

Notes: (A) Histopathologic grade (GI vs G2+G3), (B) FIGO stage (III-IV vs I-II), (C) lymph node metastasis (yes vs no), (D) clinicopathological type (S vs C), (E) DFS ( $\mathrm{H}$ vs $\mathrm{L}$ ), and $(\mathbf{F}) \mathrm{OS}(\mathrm{H}$ vs $\mathrm{L})$.

Abbreviations: ALDHI, aldehyde dehydrogenase I; OR, odds ratio; HR, hazard ratio; FIGO, International Federation of Gynecology and Obstetrics; DFS, disease-free survival; OS, overall survival; S, serous ovarian carcinoma; C, clear cell adenocarcinoma; $\mathrm{H}$, high; L, low.

presentation, rapid progression, and poor survival. Considering the high morbidity and mortality of OC, researchers have devoted themselves to identifying new prognostic markers to achieve better clinical decision-making regarding therapy and outcomes. It is well-known that ALDH1 is a CSC marker for a variety of solid tumors, including OC, and is used to enrich ovarian CSCs. ${ }^{26}$ ALDH1 is closely related to stemness of ovarian CSCs, including factors such as self-renewal, enhanced invasion, colony formation, and chemoresistance. ${ }^{27,28}$ Some researchers had successfully isolated stem-like cells from OC cell lines by ALDH1 activity and demonstrated their superior abilities in colony formation and tumorigenicity compared to ALDH1 cells in mice. ${ }^{29,30}$ Recently, Kakar et al ${ }^{31}$ showed that doxorubicin liposomes, when combined with withaferin A, elicits synergistic effects on inhibition of cell proliferation in $\mathrm{OC}$ cells and inhibits 
expression of ALDH1 protein. These observations might indicate that increasing ALDH1 expression is associated with clinicopathological features in patients with OC. Likewise, inhibiting ALDH1 expression could overcome chemoresistance in human OCs. Therefore, ALDH1 could be a potential target for OC therapy.

The association between ALDH1 expression and OC prognosis is being increasingly investigated. Ma and Zhao found that high mRNA expression of five ALDH1 isoenzymes, including ALDH1A1, ALDH1A2, ALDH1A3, ALDH1B1, and ALDH1L1, was not correlated with OS in serous cancer or endometrioid cancer patients. However, ALDH1A3's high mRNA expression is associated with worse OS in grade II OC patients. ${ }^{32}$ Apart from that, Sun et $\mathrm{al}^{16}$ also indicated that a high ALDH1 protein expression was associated with poor DFS. Nevertheless, the view from Chang et $\mathrm{al}^{25}$ was in opposition to this hypothesis. Therefore, we performed a meta-analysis to evaluate the value of ALDH1 as a prognosis marker in OC patients by summarizing all related studies.

The present meta-analysis of 18 included studies, which detected ALDH1 expression in OC tissue samples, indicated that elevated ALDH1 expression was significantly associated with poor OS but not with DFS in patients with ovarian tumor. Additionally, when considering clinicopathological features, our results show that ALDH1 was most frequently elevated in patients with poor clinicopathological characteristics, such as advanced FIGO stage, lymph node metastasis, and distant metastasis, suggesting that ALDH1 may be feasible for tumor staging and metastasis in OC. To sum up, this meta-analysis provides evidence to estimate the significance of ALDH1 detection in patients with OC.

In our meta-analysis, there exist different degrees of heterogeneity for clinicopathological characteristics and survival outcome. Therefore, subgroup analysis was conducted based on sample size and ethnicity. However, subgroup analysis of methods for FIGO stage in ethnicity reached indifferent conclusions. Elevated ALDH1 expression was significantly associated with poor stage in Asians but with early stage in non-Asians. Subgroup analysis of sample size for OS and DFS showed increasing ALDH1 expression in the $<100$ group rather than the $\geq 100$ group, which might be due to the significant heterogeneity in the $\geq 100$ group. In addition, no evidence indicated publication bias for tumor stage, tumor grade, lymph node metastasis, clinicopathological type, distant metastasis, OS, and DFS in regard to ALDH1 high expression using Begg's test. In addition, the result of the sensitivity analysis suggested that the pooled OR/HRs were stable and reliable.
There are also several limitations in this current metaanalysis, although our results are promising. First, considerable heterogeneity existed in the present study, to which we applied a relatively conservative random-effects model if there was a significant heterogeneity; therefore, the prognostic value of ALDH1 in OC may be underestimated. Second, due to the current disagreement on the cutoff value for ALDH1, our study did not provide results regarding the optimal cutoff value and whether the cutoff values differed in the assessment of clinicopathological characteristics and prognosis values. In addition, although we performed subgroup analysis based on sample size and ethnicity, other variables were different in the primary studies. The number of included studies was also limited; thus, we could not conduct in-depth subgroup analysis based on the various variables. Therefore, further large-scale multicenter studies on homogeneous patients and diagnostic methods are required to investigate the prognostic values of ALDH1 in OC. Despite the limitations of our study, our meta-analysis is the first study to demonstrate a correlation between ALDH1 and the clinicopathological characteristics and prognosis in OC.

\section{Conclusion}

Our results indicate that increasing levels of ALDH1 predict poor prognosis and clinicopathological characteristics in OC. Future studies are needed to explore tailored treatments that directly target ALDH1 for the improvement of survival in OC.

\section{Acknowledgments}

The authors would like to acknowledge all the members of Department of Oncology of Weifang Traditional Chinese Hospital. This study was supported by grants from the National Natural Science Foundation of China (Nos 81473513 and 81673799).

\section{Author contributions}

Changgang Sun was involved in the concept and design of the study. Wenge Zhao drafted the manuscript. All authors participated in acquisition, analysis, and interpretation of the data; critical revision of the manuscript; and read and approved the final version.

\section{Disclosure}

The authors report no conflicts of interest in this work.

\section{References}

1. Siegel RL, Miller KD, Jemal A. Cancer statistics, 2016. CA Cancer J Clin. 2016;66(1):7-30. 
2. Kipps E, Tan DS, Kaye SB. Meeting the challenge of ascites in ovarian cancer: new avenues for therapy and research. Nat Rev Cancer. 2013;13(4):273-282.

3. Rahman M, Deleyrolle L, Vedam-Mai V, Azari H, Abd-El-Barr M, Reynolds BA. The cancer stem cell hypothesis: failures and pitfalls. Neurosurgery. 2011;68(2):531-545.

4. Antoniou A, Hebrant A, Dom G, Dumont JE, Maenhaut C. Cancer stem cells, a fuzzy evolving concept: a cell population or a cell property? Cell Cycle. 2013;12(24):3743-3748.

5. Huang R, Li X, Holm R, Trope CG, Nesland JM, Suo Z. The expression of aldehyde dehydrogenase 1 (ALDH1) in ovarian carcinomas and its clinicopathological associations: a retrospective study. BMC Cancer. 2015;15(1):502.

6. Zhou C, Sun B. The prognostic role of the cancer stem cell marker aldehyde dehydrogenase 1 in head and neck squamous cell carcinomas: a meta-analysis. Oral Oncol. 2014;50(12):1144-1148.

7. Chute JP, Muramoto GG, Whitesides J, et al. Inhibition of aldehyde dehydrogenase and retinoid signaling induces the expansion of human hematopoietic stem cell. Proc Natl Acad Sci U S A. 2006;103(31): 11707-11712.

8. Kuroda T, Hirohashi Y, Torigoe T, et al. ALDH1-high ovarian cancer stem-like cells can be isolated from serous and clear cell adenocarcinoma cells, and ALDH1 high expression is associated with poor prognosis. PLoS One. 2013;8(6):e65158.

9. Yu L, Zhu B, Wu S, et al. Evaluation of the correlation of vasculogenic mimicry, ALDH1, KiSS-1, and MACC1 in the prediction of metastasis and prognosis in ovarian carcinoma. Diagn Pathol. 2017;12(1):23.

10. Stang A. Critical evaluation of the Newcastle-Ottawa scale for the assessment of the quality of nonrandomized studies in meta-analyses. Eur J Epidemiol. 2010;25(9):603-605.

11. Zhou ZR, Zhang TS, Li B, Mao Z, Zeng XT, Liu SX. Extracting and transforming of appropriate data of meta-analysis in survival curve. Chin J Evid Based Cardiovasc Med. 2014;6:243-247.

12. Jing H, Liu XY, Chen YL, Bai LP, Zheng A. Expression level of membrane-associated proteins numb in epithelial ovarian carcinoma and its relationship with ovarian cancer stem cell markers CD117, CD133, ALDH1. J Sichuan Univ. 2016;47(6):878-882.

13. Xue QM, Ji XQ, Wang HY. Expressions of ALDH1 and Nanog proteins in ovarian cancer and their clinical significance. Chin J Clin Res. 2015;28(8):992-995

14. Yu L, Song WQ, Zhou L, Wu SW, Wang DN. Correlation between expression of ALDH1/ABCG2 and microvessel formation in epithelial ovarian cancer. Chin J Pathophysiol. 2016;32(10):1807-1814.

15. Kim MJ, Kim AR, Jeong JY, et al. Correlation of ALDH1 and Notch3 expression: clinical implication in ovarian carcinomas. J Cancer. 2017;8(16):3331-3342.

16. Sun Y, Jia X, Wu X. High expressions of Lgr5 and ALDH1 in primary epithelial ovarian cancer correlate with advanced tumor stage and grade as well as poor prognosis of the patients. Gynecol Obstet Invest. Epub 2015 Jun 20.

17. Landen CN, Goodman BW, Nick AM, et al. Markers for discovering stemness in ovarian cancer. Gynecol Oncol. 2009;112(2):S140-S141.
18. Mizuno T, Suzuki N, Makino H, et al. Cancer stem-like cells of ovarian clear cell carcinoma are enriched in the ALDH-high population associated with an accelerated scavenging system in reactive oxygen species. Gynecol Oncol. 2015;137(2):299-305.

19. Ruscito I, Cacsire Castillo-Tong D, Vergote I, et al. Exploring the clonal evolution of CD133/aldehyde-dehydrogenase-1 (ALDH1)-positive cancer stem-like cells from primary to recurrent high-grade serous ovarian cancer (HGSOC). A study of the ovarian cancer therapyinnovative models prolong survival (OCTIPS) consortium. Eur $J$ Cancer. 2017;79:214-225.

20. Chen PX, Li QY, Yang Z. Musashi-1 expression is a prognostic factor in ovarian adenocarcinoma and correlates with ALDH-1 expression. Pathol Oncol Res. 2015;21(4):1133-1140.

21. Deng S, Yang X, Lassus H, et al. Distinct expression levels and patterns of stem cell marker, aldehyde dehydrogenase isoform 1 (ALDH1), in human epithelial cancers. PLoS One. 2010;5(4):e10277.

22. Liebscher CA, Prinzler J, Sinn BV, et al. Aldehyde dehydrogenase 1/epidermal growth factor receptor coexpression is characteristic of a highly aggressive, poor-prognosis subgroup of high-grade serous ovarian carcinoma. Hum Pathol. 2013;44(8):1465-1471.

23. Wang YC, Yo YT, Lee HY, et al. ALDH1-bright epithelial ovarian cancer cells are associated with CD44 expression, drug resistance, and poor clinical outcome. Am J Pathol. 2012;180(3):1159-1169.

24. Ayub TH, Keyver-Paik MD, Debald M, et al. Accumulation of ALDH1positive cells after neoadjuvant chemotherapy predicts treatment resistance and prognosticates poor outcome in ovarian cancer. Oncotarget. 2015;6(18):16437-16448.

25. Chang B, Liu G, Xue F, et al. ALDH1 expression correlates with favorable prognosis in ovarian cancers. Mod Pathol. 2009;22(6):817-823.

26. Park YT, Jeong JY, Lee MJ, et al. MicroRNAs overexpressed in ovarian ALDH1-positive cells are associated with chemoresistance. J Ovarian Res. 2013;6(1):18

27. Wu D, Wang J, Cai Y, et al. Effect of targeted ovarian cancer immunotherapy using ovarian cancer stem cell vaccine. J Ovarian Res. 2015; 8:68.

28. Ginestier C, Hur MH, Charafe-Jauffret E, et al. ALDH1 is a marker of normal and malignant human mammary stem cells and a predictor of poor clinical outcome. Cell Stem Cell. 2007;1(5):555-567.

29. Wu XH, Zhao TT, Cao QY, et al. Effect of low-dose metronomic chemotherapy on ALDH1 expression in human ovarian carcinoma xenograft tumor in nude mice. Tumor. 2015;35(4):377-383.

30. Yasuda K. The significance of stress-responsive molecules in cancer stem cells derived from ovarian and endometrial cancer. Cancer Res. 2012;72(8).

31. Kakar SS, Worth CA, Wang Z, Carter K, Ratajczak M, Gunjal P. DOXIL when combined with Withaferin A (WFA) targets ALDH1 positive cancer stem cells in ovarian cancer. EMBO Mol Med. Epub 2016 Apr 19;4.

32. Ma YM, Zhao S. Prognostic values of aldehyde dehydrogenase I isoenzymes in ovarian cancer. Onco Targets Ther. 2016;9:1981-1988.
OncoTargets and Therapy

\section{Publish your work in this journal}

OncoTargets and Therapy is an international, peer-reviewed, open access journal focusing on the pathological basis of all cancers, potential targets for therapy and treatment protocols employed to improve the management of cancer patients. The journal also focuses on the impact of management programs and new therapeutic agents and protocols on

\section{Dovepress}

patient perspectives such as quality of life, adherence and satisfaction. The manuscript management system is completely online and includes a very quick and fair peer-review system, which is all easy to use. Visit http://www.dovepress.com/testimonials.php to read real quotes from published authors. 